\title{
New possibilities of graphics software in the analysis of thermograms of patient's lower limbs - a technical note
}

\author{
Monika WIDEŁa, a, ${ }^{\text {, }}$, Sławomir GRZEGORCZYN ${ }^{a}$ \\ ${ }^{a}$ Department of Biophysics, School of Medicine with the Division of Dentistry in Zabrze, Medical University of Silesia, 19 H. \\ Jordan Str., 41-808 Zabrze, Poland \\ ${ }^{b}$ Department of Surgery, Provincial Specialist Hospital No. 3 in Rybnik; 46 Energetyków, 44-200 Rybnik, Silesia, Poland \\ *E-mail address: moniczka@ipnet.pl
}

\begin{abstract}
The analysis involved thermograms of the lower limbs of a patient suffering from symptomatic L-S segment discopathy with spinal root compression syndrome (symptomatic lumbar discopathy) qualified for surgical treatment. The thermograms were obtained using the Flir Ebx 50 camera. They were developed with the use of the software included with the Flir Ebx 50 camera, as well as the Origin Pro 2020 data analysis and graphing software. ROIs (Regions of Interest) were specified and analyzed in terms of temperature (average, maximum or minimum) temperature distributions, isotherms, and specified surface areas limited by selected isotherms. According to the analysis of the thermograms, the images obtained with Origin Pro 2020 enable a more advanced presentation of the temperature distribution, by taking into account the isotherms with selected temperatures and by calculating the area limited by a given isotherm (or between two isotherms) they allow to introduce an additional surface parameter related to specific isotherms. It provides additional information (parameter) in comparison with analogous ROIs on healthy and pathologic limbs in the same patient.
\end{abstract}

Key words: thermography; lumbar discopathy; Origin pro.

\section{Introduction}

Thermography involves the process of recording and processing digital images obtained in the infrared radiation range. It gives the possibility to analyze information about the temperature distribution on the surface of a tested object or a set of objects. The application of these tests in medicine is used to assess the temperature distribution on the surface of the human body. ${ }^{1}$ Visualization of the distribution of the emitted infrared radiation, and thus the distribution of surface temperatures, allows for obtaining and analyzing information on the processes taking place on the skin surface and in the subcutaneous tissues (both physiological and pathological), ${ }^{2}$ which result in changes in the skin surface temperatures. ${ }^{3}$ Thermographic examinations are used more and more often in medical sciences, ${ }^{4,5}$ especially in oncologic examinations ${ }^{6}$ and in the case of breast cancer. ${ }^{7,8}$ Temperature changes observed in the lower extremities of the thermal skin map are associated with a healthy state of blood supply which might be connected with blood stasis, inflammatory states, and swelling that occurs in the soft tissues. ${ }^{9}{ }^{10}$ The importance of the use of thermographic tests increased in the time of the COVID-19 pandemic. $.^{11},{ }^{12}$

Due to the scope of our studies, we are interested in the use of thermographic studies in neurology ${ }^{13},{ }^{14}$ and neurosurgery ${ }^{15}$ in the imaging process of neuromuscular diseases. ${ }^{16}$ An interesting application of thermography is a diagnosis of CTS by thermal imaging in both hands from phalanges to the area of the wrist on the external and palmar side of the palm. ${ }^{17}$ Back pain is the most common cause of absence from work globally. ${ }^{18}$ Thermography has become a tool in imaging root pain syndromes, neuropathies, and in patients who suffered from strokes. ${ }^{19}$ What is more, thermograms are used in the case of lumbosacral discopathy and other spinal diseases. ${ }^{20}$ Serious diseases (neoplastic changes, metastases, infections) are rarely the cause of back pain, most often the pathology is associated with discopathy. ${ }^{21}$ In over $90 \%$ of cases, properly conducted pharmacotherapy and rehabilitation allow to control the pain associated with lumbar discopathy. Clinically observed symptoms of tendon reflexes weakening, stretching symptoms (including the Laseque's symptom) and weakening of muscle strength in the affected limb (correlating with the MR) constitute indications for surgical treatment if they persist for a long time despite conservative treatment. $^{22}$ Scientific reports show that patients with symptomatic discopathy often suffer from lower limb thermal disorders understood as the difference in temperature distribution on the affected limb as compared with the healthy limb. ${ }^{23}$ 
In this article, we have presented a case report of a patient qualified for surgery based on clinical indications and imaging tests. The analyzed case presents the possibility of extending visualization of temperature data from thermograms by using professional data analysis and graphing software Origin Pro 2020. The program allows for a presentation of isotherms for selected temperatures and the surface area limited by an indicated isotherm or between two isotherms.

\section{Purpose}

Showing the possibility of using the Origin Pro program as a tool for accurate imaging of the temperature distribution on the lower limbs in a patient qualified for surgery.

Summary and comparison of thermograms obtained for the same patient from the Flir Ebx 50 Camera, using the original Flir camera software and after exporting the thermogram to a .csv file, which was subsequently imported to Origin Pro.

Processing of the obtained data in order to show new possibilities of analyzing the surface temperature distribution and obtaining new, in terms of quality, interpretations of the studied ROIs.

\section{Material and method}

The analysis involved lower limb thermograms of a 21-year-old female patient suffering from symptomatic L-S segment discopathy with spinal root compression syndrome, with a weight of $77 \mathrm{~kg}$ and height of $165 \mathrm{~cm}(\mathrm{BMI}=28.3)$. The patient reported pain in the lower limbs (around calves and shins, persisting for over 6 months) before hospitalization and qualification for surgery (fenestration). The patient has been smoking $1 / 2$ pack of cigarettes a day for about 3 years. Due to depression, the patient takes Asertin and is under constant care of a specialist from a mental health clinic. Based on imaging tests (MRI) and clinical examination, she has been qualified for surgical treatment of lumbar discopathy.

Thermograms of the lower limbs were taken before the surgery, the scanning session consisted of eight photographs of the following areas: hip, knee, and ankle joints, and a holistic image of the lower limbs in a front and back projection. These photos do not allow for the identification of the examined person. In order to ensure the stability of the experiment conditions, the photos were taken in a room with a fixed temperature of $22^{\circ} \mathrm{C}$. Thermograms were performed at a distance of $1.5 \mathrm{~m}$ from the patient and at the height of the camera at the patient's knee level. Before taking the thermograms the patient remained in the room for 10 minutes.

The software for developing thermograms from a Flir Camera makes it possible to select an ROI (Region of Interest) of a specific shape (rectangle, ellipse, etc.). Subsequently, maximum, minimum, and average temperatures may be determined for the specific region. Thermograms developed with the Flir Camera software do not distinguish isotherms in the form of lines connecting points of the same temperature. For this reason, as well as in order to obtain a better quality of the thermal images of the examined limbs, we decided to use the Origin Pro 2020 data analysis and graphing software. A selected thermogram was exported to a .csv file by means of the Flir Camera software and, subsequently, the obtained .csv file was imported to Origin Pro 2020. Thermograms developed with the Origin Pro 2020 program allow, among other things, to visualize specific, selected isotherms on the thermogram, and to calculate the surface area of the region limited by a selected isotherm or a region between specified isotherms. As a result, it is possible to introduce new parameters, such as surfaces limited by isotherms, allowing their comparison in the corresponding ROIs on the healthy and diseased limb (before and after the procedure). This has provided new possibilities for interpreting thermograms. Each type of analysis is configurable, and the user decides how to analyze a thermogram. Hence, Origin Pro 2020 allows you to expand the scope of comparative analyzes, offering additional parameters that can be used in descriptive statistics of the obtained thermograms.

\section{Results and commentary}

The analysis involved lower limb thermograms of a female patient suffering from symptomatic L-S segment discopathy with spinal root compression syndrome, with a weight of $77 \mathrm{~kg}$ and height of $165 \mathrm{~cm}(\mathrm{BMI}=28.3)$. The thermograms were obtained using the Flir Ebx 50 camera. These were selected thermograms of the lower limbs obtained in the same shot before the procedure (a). Figure 1 shows examples of thermograms prepared with the software included with the Flir Ebx 50 camera. One of them (1a) shows an ROI in the form of an ellipse with a specification of temperatures in this region: maximum, minimum, and average for the ROI.

Thanks to the scale provided on the right side of a thermogram, they allow listing regions with a higher temperature. Having indicated an ROI, it is possible to specify selected temperatures in this region as output parameters for statistical processing of data for a study group of patients. The next step in the data processing was an attempt to obtain isotherms in the test regions. Therefore, after exporting thermograms from the Flir camera software to .csv files, they were subsequently imported to Origin Pro 2020 for further analysis. The following images show the thermograms from Figure 1 processed by Origin Pro 2020. Figure 2a shows an example isotherm thermogram and the colors correspond to regions assigned to individual temperature ranges presented on the scale on the right side of the thermogram, exclusive of areas with temperatures higher than $33^{\circ} \mathrm{C}$ or lower than $26^{\circ} \mathrm{C}$ (marked in white).

Figure 2a proves that Origin Pro 2020 allows you to highlight isotherms on thermograms and specify areas between the isotherms with corresponding colors, according to color map templates or specifically defined colors for these areas. It is possible to determine the temperature value for isotherms as 
well as their number on the thermogram. Thanks to the option to exclude regions with temperatures below (and above) a defined point (white fields on the thermogram), it is possible to focus on areas with a selected temperature range. Figure $\mathbf{2 b}$ shows the same thermogram on which isotherms with three different temperatures have been listed. This type of isotherm distinction allows for selective analysis of the temperature distribution on the test regions. What is more, thermogram $\mathbf{2} \mathbf{b}$ shows regions on the lower part of the leg, with temperature exceeding $32^{\circ} \mathrm{C}$, as marked in gray. The thermogram prepared with the Origin Pro

(a)

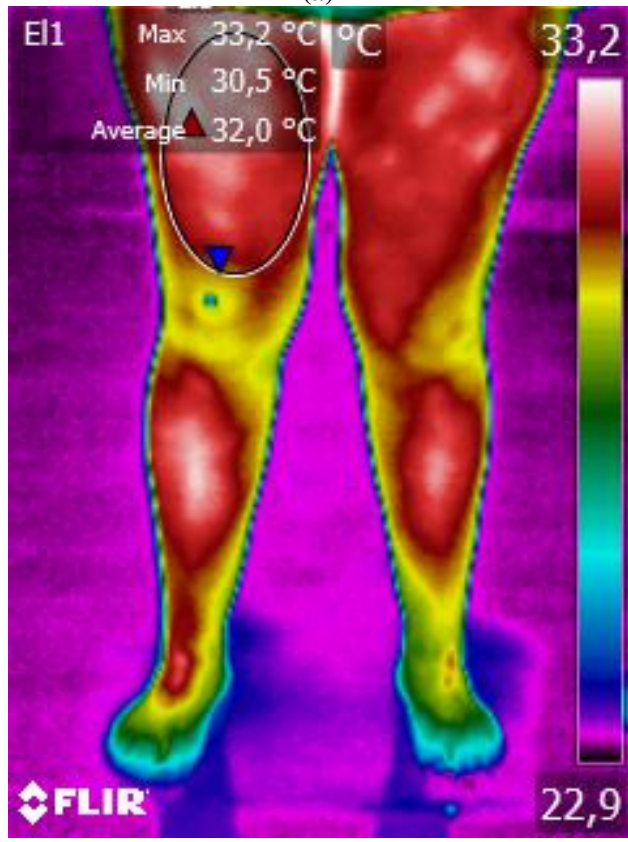

software was the basis for designating surface areas of these regions. The designated surfaces, in this case for temperatures above $32^{\circ} \mathrm{C}$, may constitute an additional parameter in a comparative analysis of similar regions, e.g., on the lower limb. By selecting surfaces limited with two isotherms and then subtracting them, you can obtain a surface with temperatures between these temperatures. We think these may constitute additional parameters introduced to statistical comparative analysis. (b)

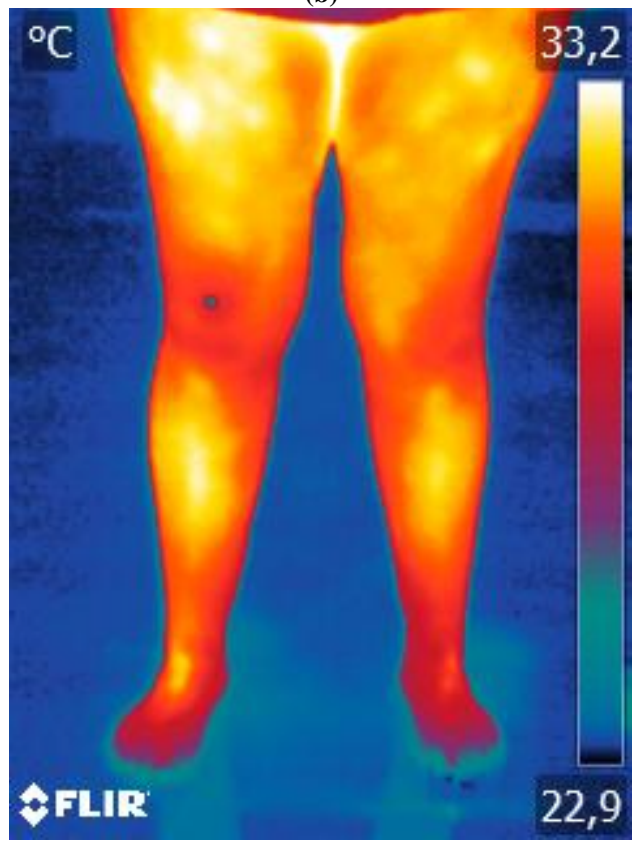

Figure 1. Source thermogram obtained with the FLIR camera, Rainbow HC projection (a), marked regions of maximum, minimum and average temperatures, and Arctic projection (b)

(a)

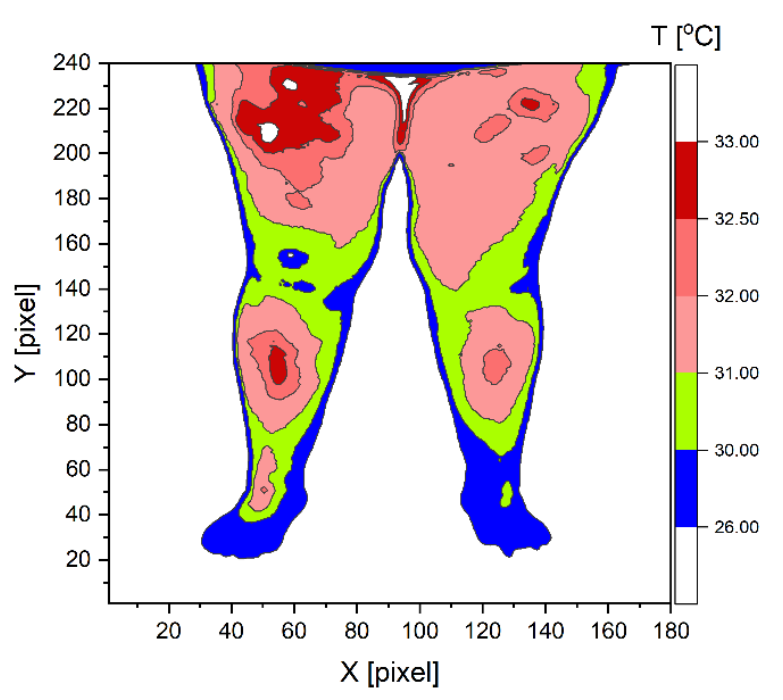

(b)

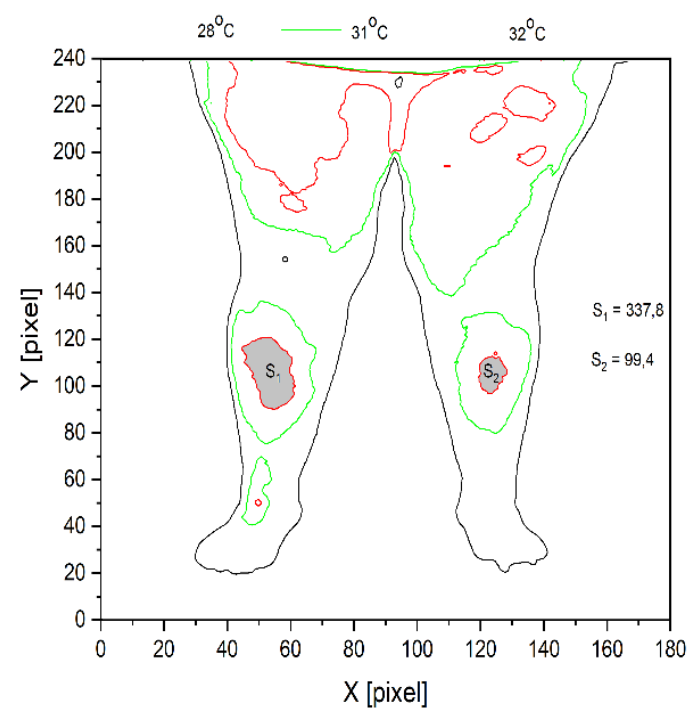

Figure 2. The lower limb thermogram from Figure 1 processed by Origin Pro 2020, with visualized isotherms and a color map for each temperature range (a) and with selected isotherms and areas on the lower leg with temperatures exceeding $32^{\circ} \mathrm{C}$, marked in gray (b). 
The Flir Camera software also enables an analysis of the average temperature (maximum or minimum) over a selected section. In turn, a thermogram prepared in Origin Pro provides for a possibility of visualization of the course of temperature changes in the selected direction, as marked with a line on the thermogram. This has been shown in Figure 3.

As shown in Figure 3, the thermal sections show temperature distributions along with the selected directions vertically (to the right of the thermogram) and horizontally (above the thermogram). The lines along which the temperature distributions are imaged have been marked on the thermogram with the corresponding chart colors. These lines can be moved on the thermogram in a direction perpendicular to the line. It is also possible to place a diagonal line along which temperature distributions are presented, as well as to highlight a section on which the temperature distribution is to be analyzed. What is more, it is possible to select a region between, e.g. two horizontal lines and determine the average (maximum and minimum) temperature for these regions calculated in the selected region along the vertical section. The graph presented above the thermogram in Figure 4 shows average temperatures along the vertical section, limited by blue horizontal lines for the entire thermogram (a) and the enlarged areas of the left (b) and right (c) shin.
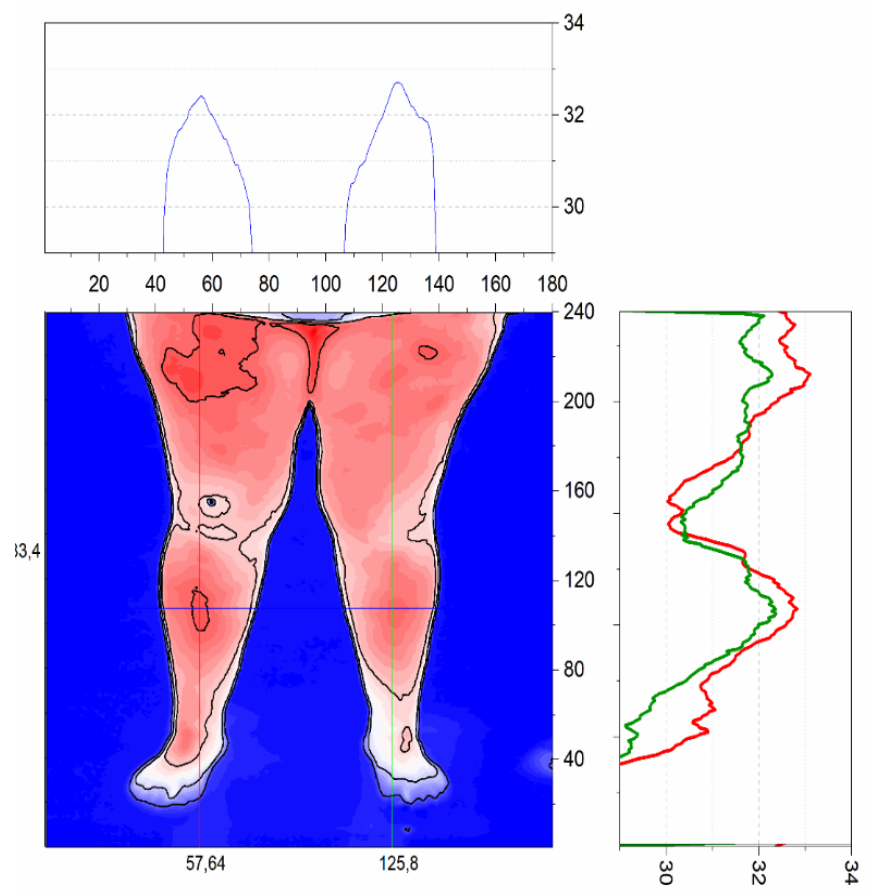

Figure 3. Thermogram from Figure 1 developed in Origin Pro, illustrating the thermal cross-sections in the selected directions (color palette Thermometer). (a)

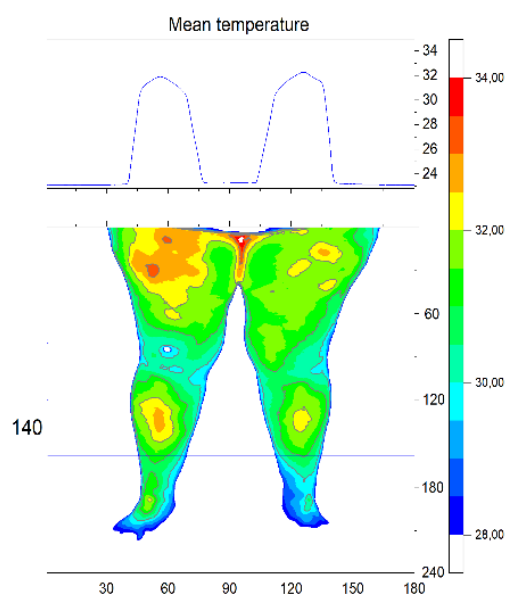

(b)

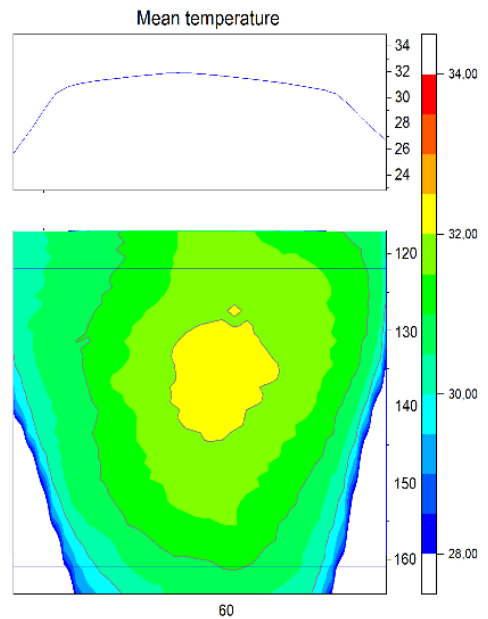

(c)

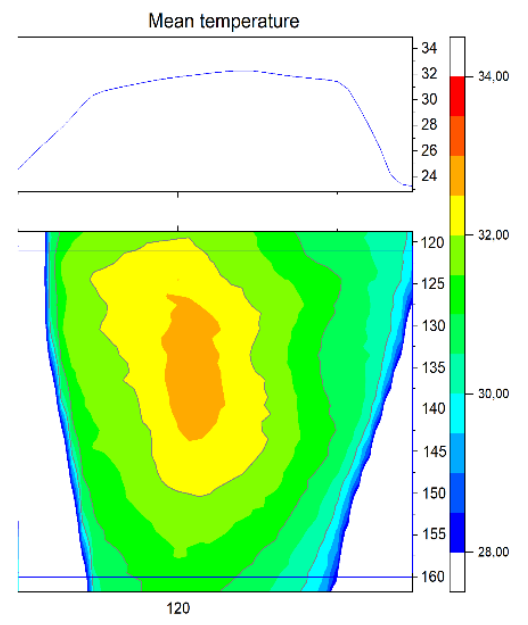

Figure 4. The thermogram from Figure 1 after processing in Origin Pro 2020, with thermal cross-sections on the lower leg (mean temperatures in the vertical direction of the section between the blue lines) (a) and in the enlarged area of the right (b) and left (c) shin.

In Figure 4, the width of the horizontal strip of the analyzed temperature (averaged vertically between the blue lines) was 40 pixels, the center of the belt being fixed on a horizontal line of 140 pixels. The graphs presented above the thermograms show the change in the average temperatures in this strip along the level. Similar stripes of analysis can be selected for a vertical strip. Origin Pro 2020 also enables the specification of changes in maximum or minimum temperatures within a selected strip. In addition, as indicated earlier (Figure 2b), it is possible to designate regions limited by individual isotherms, fixed in advance for specific temperatures. For example, for Figure 4c, the following regions have been selected: with temperatures exceeding $33^{\circ} \mathrm{C}$ (brown field): $\mathrm{S}_{\mathrm{t}}>33=74.1 \mathrm{px}$; with temperatures exceeding $32^{\circ} \mathrm{C}$ (brown + yellow field): $\mathrm{S}_{\mathrm{t}>32.5}=337,8 \mathrm{px}$; and after subtracting fields: $\mathrm{S}_{\mathrm{t}>32.5}-\mathrm{S}_{\mathrm{t}>33}$ the resulting region field has temperatures between 32.5 a $33^{\circ} \mathrm{C}$ : $\mathrm{S}_{32.5<\mathrm{t}<33}=\mathrm{S}_{\mathrm{t}>32.5}-\mathrm{S}_{\mathrm{t}>33}=263.7 \mathrm{px}$.

Similarly, any surfaces between isotherms with pre-defined temperatures can be obtained from the thermogram. By placing on the imaged surface, a rectangle of certain sizes, made from a 
material clearly visible in infrared $(1 \mathrm{~cm} \times 1 \mathrm{~cm}$ aluminum foil rectangle located on the right knee), you can assign the rectangle surface with several pixels on the thermogram to enable calculation of surface units expressed in pixels per surface area expressed in $\mathrm{cm}^{2}$. Zooming in on selected thermogram fragments enables a comparison of selected ROIs more accurately and visualization of differences between corresponding regions.

\section{Conclusions}

The use of Origin Pro 2020 on the selected example enabled a new representation of thermograms from the thermal imaging camera to obtain additional information (as well as parameters) related to the temperature distribution on the patient's lower limbs.

The advantage of the thermograms prepared in Origin Pro 2020 is the imaging of the course of isotherms with selected temperatures on the thermogram and exclusion of regions with temperatures below (and/or above) the range of our interest.
Moreover, it is possible to calculate the surface area with temperatures exceeding a specific level or the surface area in the ROI with temperatures between specified temperatures. The areas thus determined may be taken into account as variables in the statistical comparative analysis in a test group.

With the use of Origin Pro 2020, a statistical comparative analysis of surgical treatment outcomes in a patient's group could be expanded. The weaknesses of this method include the fact that moving thermograms between programs and preparing thermograms in Origin Pro 2020 is time-consuming.

\section{Acknowledgments}

This work was supported by the project of MUS (Medical University of Silesia): KNW-1-018/N/9/I.

Bioethics commission number: Resolution no. KNW/0022/KB1/10/I/18.

\section{References}

1. Lasanen R. Infrared Thermography in the Evaluation of Skin Temperature: Applications in Musculoskeletal Conditions. Dissertations in Forestry and Natural Sciences No 186. University of Eastern Finland; 2015. Accessed May $12,2021$. https://erepo.uef.fi/handle/123456789/15360

2. Tanda G. The use of infrared thermography to detect the skin temperature response to physical activity. J Phys Conf Ser. 2015;655:12062. https://doi.org/10.1088/1742-6596/655/1/012062

3. Staszak K. Zastosowanie termografii w monitorowaniu procesu leczenia urazów twarzowej części czaszki. In: Asienkiewicz R, Markocka-Mączka K, Biskup M, eds. Zdrowie publiczne standardem dobrostanu. Wydawnictwo Naukowe NeuroCentrum; 2018:249257.

4. Ivanitskii G. Thermovision in medicine. Her Russ Acad Sci. 2006;76:44-53. https://doi.org/10.1134/S1019331606010060

5. Lahiri BB, Bagavathiappan S, Jayakumar T, Philip J. Medical applications of infrared thermography: A review. Infrared Phys Technol. 2012;55(4):221-235. https://doi.org/10.1016/J.INFRARED.2012.03.007

6. Cohen Y, Dekel BZ, Krouk E, Blaunstein N. Method of Infrared Thermography for Earlier Diagnostics of Gastric Colorectal and Cervical Cancer. EC Gastroenterology and Digestive System; 2019;10:916-933.

7. Kolarić D, Herceg Z, Nola IA, et al. Thermography--a feasible method for screening breast cancer? Coll Antropol. 2013;37(2):583588.

8. Alikhassi A, Hamidpour SF, Firouzmand M, Navid M, Eghbal M. Prospective comparative study assessing role of ultrasound versus thermography in breast cancer detection. Breast Dis. 2018;37:191-196. https://doi.org/10.3233/BD-180321

9. Cholewka A, Kajewska J, Marek K, Sieroń-Stołtny K, Stanek A. How to use thermal imaging in venous insufficiency? J Therm Anal Calorim. 2017;130(3):1317-1326. https://doi.org/10.1007/s10973-017-6141-7

10. Cholewka A, Stanek A, Klimas A, Sieroń A, Drzazga Z. Thermal imaging application in chronic venous disease: Pilot study. J Therm Anal Calorim. 2014;115(2):1609-1618. https://doi.org/10.1007/s10973-013-3356-0

11. Enforcement Policy for Telethermographic Systems During the Coronavirus Disease. Food and Drug Administration (FDA); 2020. Accessed May 12, 2021. https://www.fda.gov/regulatory-information/search-fda-guidance-documents/enforcement-policytelethermographic-systems-during-coronavirus-disease-2019-covid-19-public-health

12. Silvino V, Galan S, Moreira D, Santos M. Identifying febrile humans using infrared thermograph screening: possible applications during COVID-19 outbreak. Rev Context Saúde. 2020;20(38):5-9. https://doi.org/10.21527/2176-7114.2020.38.5-9

13. Neves E, Alves J, Rosa C, Reis V. Thermography in Neurologic Practice. Open Neurol J. 2015;9:24-27. https://doi.org/10.2174/1874205X01509010024

14. Cholewka A, Drzazga Z, Sieroń A, Stanek A. Thermovision diagnostics in chosen spine diseases treated by whole body cryotherapy. J Therm Anal Calorim. 2010;102(1):113-119. https://doi.org/10.1007/s10973-010-0873-y 
15. Fraerman AP, Kolesov SN, Likhterman LB. Diagnostic possibilities and prospects of thermovision in the neurosurgical clinic. Zhurnal Vopr Nejrokhirurgii Im NN Burdenko. 1978;42(2):27-35.

16. Harun-Ar-Rashid. Importance of thermovision in radial mononeuropathy of compressive and ischaemic origin. Bangladesh Med Res Counc Bull. 1989;15(2):60-66.

17. Baic A, Kasprzyk T, Rzany M, et al. Can we use thermal imaging to evaluate the effects of carpal tunnel syndrome surgical decompression? Med (United States). 2017;96(39). https://doi.org/10.1097/MD.0000000000007982

18. Bernstein IA, Malik Q, Carville S, Ward S. Low back pain and sciatica: summary of NICE guidance. BMJ. $2017 ; 356$. https://doi.org/10.1136/bmj.i6748

19. Polidori G, Kinne M, Mereu T, Beaumont F, Kinne M. Medical Infrared Thermography in back pain osteopathic management. Complement Ther Med. 2018;39:19-23. https://doi.org/10.1016/J.CTIM.2018.05.010

20. Ammer K. Thermography in Spinal Disorders- A narrative Review. Thermol Int. 2010;20:117-125.

21. Verhagen AP, Downie A, Popal N, Maher C, Koes BW. Red flags presented in current low back pain guidelines: a review. Eur Spine J. 2016;25(9):2788-2802. https://doi.org/10.1007/s00586-016-4684-0

22. Greenberg MS. Lumbar disc herniation and lumbar radiculopathy. In: Handbook of Neurosurgery. Eighth Edi. New York: Thieme; 2016:1046-1061.

23. Gillström P. Thermography in low back pain and sciatica. Arch Orthop Trauma Surg. 1985;104(1):31-36. https://doi.org/10.1007/BF00449954 